\title{
Propagation of laser beam parameters through pure phase transmittances
}

\author{
G. Piquero, P.M. Mejías, R. Martínez-Herrero \\ Departamento de Óptica, Facultad de Ciencias Físicas, Universidad Complutense. 28040 Madrid, Spain
}

Received 11 December 1995; accepted 7 March 1996

\begin{abstract}
The propagation laws of the intensity moments of a laser beam through $A B C D$ optical systems are generalized to include pure phase transmittances. This is done by representing the behaviour of such transmittances by means of a $4 \times 4$ matrix, $\mathbf{M}$, which can be handled, to some extent, as the $A B C D$-matrices associated with ordinary first-order optical systems. This formalism enables the application of $A B C D$ propagation formulae to cascaded optical systems containing pure phase transmittances. Matrix $\mathbf{M}$ is used to determine the intensity moments at the output of two special quartic phase transmittances, namely, a circular spherically aberrated lens and a pair of orthogonal cylindrical (also aberrated) lenses.
\end{abstract}

\section{Introduction}

The characterization of spatial profiles of laser beams by means of suitable quality parameters and the description of their propagation through optical systems are topics of current interest [1-5]. One way to improve the beam quality (defined in terms of the so-called moments of both the intensity and the radiant intensity of the field) is by using soft-edge Gaussian apertures. However, in such a case, the output heam intensity might be drastically reduced. To maintain the total power without hard-edge diffraction effects, a method based on the use of a pure-phase transmittance (PPT) seems to be more appropriate. In fact, the phase function that should be employed to achieve the minimum value (highest quality) of the beam quality parameter of symmetric-intensity beams has been determined [6]. Moreover, it has recently been shown [7], that spherical aberrations (or more generally, quartic phase distortions) could improve the beam quality. Unfortunately, PPTs are not, in general, first-order optical systems. It thus seems that a simple $A B C D$-propagation law cannot be applied to this kind of transmittance. This is of special relevance when the beam travels through a cascaded optical system containing an arbitrary collection of PPTs. So far, such cascaded optical elements could not be characterized by an overall $A B C D$ matrix. This generates lengthy and cumbersome calculations of the spatial moments of the output beam, especially in the tridimensional case.

The aim of the present paper is to overcome this difficulty. Thus, to determine all first- and second-order intensity moments of the beam after travelling through a PPT, we will represent in the next section the behaviour of this transmittance by means of a $4 \times 4$ matrix, which can formally be handled, to some extent, as the matrices associated with ordinary $A B C D$ optical systems. In Section 3, as a simple application of the above formalism, we will obtain all second-order intensity moments of two special but important cases of quartic phase transmittances: (i) a circular spherically 
aberrated lens; and (ii) a pair of orthogonal cylindrical (also aberrated) lenses. Finally, some demonstrations are shown in the Appendix.

\section{Matricial representation of PPTs}

As usual, let us begin with the Wigner distribution function (WDF) $h(x, y, u, v ; z)$ of the field, defined as [8]

$$
\begin{aligned}
& h(\boldsymbol{\rho}, \boldsymbol{\eta} ; z)=\int \mathrm{d}^{2} s \Gamma(\boldsymbol{\rho}+\boldsymbol{s} / 2, \boldsymbol{\rho}-\boldsymbol{s} / 2 ; z) \\
& \quad \times \exp (i k \boldsymbol{\eta} \boldsymbol{s}),
\end{aligned}
$$

where $k$ is the wavenumber, $\Gamma$ denotes the crossspectral density function of a field, $z$ indicates the direction of propagation, $\boldsymbol{\rho}=(x, y), x$ and $y$ being the transversal variables, and $\boldsymbol{\eta}=(u, v)$, with $u$ and $v$ representing the angles of propagation (without taking the evanescent waves into account). Note that $(k u, k v)=$ $\left(k_{x}, k_{y}\right)$ are the wavevector components along the $x$ and $y$-axes, respectively. The dummy variable $s$ appears in the function $\Gamma\left(x_{1}, x_{2}\right)$, where $x_{i}, i=1,2$, denotes the bidimensional transversal position vectors, as a consequence of a change of variables $x_{1}-x_{2}=s$, $\boldsymbol{\rho}=\left(\boldsymbol{x}_{1}+\boldsymbol{x}_{2}\right) / 2$ required to write Eq. (1). Integration of $h$ over the angular and spatial variables then gives, respectively, the beam irradiance and (a proportionality factor apart) the radiant intensity of the field. Averages (also called intensity moments) of the function $h$, denoted by angle brackets, can be introduced as follows

$$
\begin{aligned}
\langle q\rangle & =\frac{1}{P_{i}} \int \mathrm{d} x \mathrm{~d} y \mathrm{~d} u \mathrm{~d} v q(x, y, u, v) \\
& \times h(x, y, u, v ; z),
\end{aligned}
$$

where $P_{\mathrm{i}}=\int \mathrm{d} x \mathrm{~d} y \mathrm{~d} u \mathrm{~d} v h(x, y, u, v ; z)$ is the total power (zero-order moment) and $q$ represents some function of the spatial and/or angular variables. As is well known, if we assume, for simplicity, that $\langle x\rangle=$ $\langle y\rangle=\langle u\rangle=\langle v\rangle=0$, the moments $\left\langle x^{2}+y^{2}\right\rangle$ and $\left\langle u^{2}+v^{2}\right\rangle$ can be understood, respectively, as a measure of the (squared) width of the bearn at plane $z$ and of its associated (squared) far-field divergence. Other moments, such as $\langle x u\rangle,\langle y u\rangle,\langle x y\rangle,\langle u v\rangle$, etc. are related to the characteristics of asymmetry, astigmatism and spatial orientation of the beam [4,9].
To handle tridimensional beams propagating through $A B C D$ systems, it is useful to define two matrices, namely,

$\mathbf{F}=\left(\begin{array}{c}\langle x\rangle \\ \langle y\rangle \\ \langle u\rangle \\ \langle v\rangle\end{array}\right)$,

and the so-called beam matrix [4]

$\mathbf{S}=\left(\begin{array}{cccc}\left\langle x^{2}\right\rangle & \langle x y\rangle & \langle x u\rangle & \langle x v\rangle \\ \langle x y\rangle & \left\langle y^{2}\right\rangle & \langle y u\rangle & \langle y v\rangle \\ \langle x u\rangle & \langle y u\rangle & \left\langle u^{2}\right\rangle & \langle u v\rangle \\ \langle x v\rangle & \langle y v\rangle & \langle u v\rangle & \left\langle v^{2}\right\rangle\end{array}\right)$,

which can also bc written in the form

$\mathbf{F}=\frac{1}{P_{\mathrm{i}}} \int \hat{p} h(x, y, u, v ; z) \mathrm{d} x \mathrm{~d} y \mathrm{~d} u \mathrm{~d} v$,

and

$\mathbf{S}=\frac{1}{P_{\mathrm{i}}} \int \hat{p} \hat{p}^{t} h(x, y, u, v ; z) \mathrm{d} x \mathrm{~d} y \mathrm{~d} u \mathrm{~d} v$,

where $\hat{p}^{t} \equiv(x, y, u, v)$, the symbol $t$ denoting the transposed matrix. Taking Eqs. (5) and (6) into account, matrices $\mathbf{F}$ and $\mathbf{S}$ at the output of an arbitrary $A B C D$ optical system (characterized by a $4 \times 4$ matrix $\mathbf{M})$ are given in terms of the WDF of the beam at the entrance plane of the system, $h_{\mathrm{i}}(x, y, u, v ; z)$, through the equations

$\mathbf{F}_{\mathrm{o}}=\frac{1}{P_{\mathrm{i}}} \int \mathbf{M} \hat{p} h_{\mathrm{i}}(x, y, u, v ; z) \mathrm{d} x \mathrm{~d} y \mathrm{~d} u \mathrm{~d} v$,

and

$\mathbf{S}_{\mathrm{o}}=\frac{1}{P_{\mathrm{i}}} \int \mathbf{M} \hat{p} \hat{p}^{t} \mathbf{M}^{t} h_{\mathrm{i}}(x, y, u, v ; z) \mathrm{d} x \mathrm{~d} y \mathrm{~d} u \mathrm{~d} v$,

where the subscript "o" denotes the output plane of the $A B C D$ system.

Let us now consider a thin PPT characterized by a transmittance function $t(x, y)=\exp [i k \phi(x, y)]$. Accordingly, the complex amplitude of the beam, $g(x, y)$, atter crossing the PPT, will be given in terms of the value of the input complex field, $f(x, y)$, as follows

$g(x, y)=\exp [i k \phi(x, y)] f(x, y)$.

We will next show that the propagation laws of the first and second-order moments (Eqs. (7) and (8)) 
are also valid for PPTs provided that we describe the behaviour of such transmittances by means of the following matrix:

$$
\mathbf{M}_{\mathrm{PPT}}=\left(\begin{array}{ll}
\mathbf{I} & \mathbf{0} \\
\mathbf{C} & \mathbf{I}
\end{array}\right) \text {, }
$$

I being the $2 \times 2$ identity matrix, and

$\mathbf{C}=\left(\begin{array}{cc}\frac{1}{x} \frac{\partial \phi}{\partial x} & 0 \\ 0 & \frac{1}{y} \frac{\partial \phi}{\partial y}\end{array}\right)$.

To prove the validity of Eq. (7) it suffices to note that (see Eq. (9)) [12]

$$
\begin{aligned}
\langle u\rangle_{\mathrm{o}} & =\frac{1}{i k P_{\mathrm{i}}} \int\left(\frac{\partial g}{\partial x}\right) g^{*} \mathrm{~d} x \mathrm{~d} y \\
& =\frac{1}{i k P_{\mathrm{i}}} \int \frac{\partial f}{\partial x} f^{*} \mathrm{~d} x \mathrm{~d} y+\frac{1}{P_{\mathrm{i}}} \int \frac{\partial \phi}{\partial x}|f|^{2} \mathrm{~d} x \mathrm{~d} y \\
& =\langle u\rangle_{\mathrm{i}}+\left\langle\frac{\partial \phi}{\partial x}\right\rangle_{\mathrm{i}},
\end{aligned}
$$

where the subscripts "o" and " $\mathrm{i"} \mathrm{again} \mathrm{refer,} \mathrm{respec-}$ tively, to the complex amplitude of the beam after and before crossing the PPT. In a similar way it can be shown that

$\langle v\rangle_{\mathrm{o}}=\langle v\rangle_{\mathrm{i}}+\frac{1}{P_{\mathrm{i}}} \int \frac{\partial \phi}{\partial y}|f|^{2} \mathrm{~d} x \mathrm{~d} y$.

Finally, since $\langle x\rangle$ and $\langle y\rangle$ are intensity moments of spatial variables only, they do not change under propagation through PPTs. The propagations law (Eq. (7)) is thus fulfilled. To prove now the applicability of Eq. (8) to this kind of transmittance let us first calculate the elements of the $4 \times 4$ matrix $\mathbf{T} \equiv \mathbf{M}_{\mathrm{PPT}} \hat{p} \hat{p}^{t} \mathbf{M}_{\mathrm{PPT}}^{t}$ appearing inside the integral of Eq. (8). After some algebra we find

$$
\begin{aligned}
& t_{11}=x^{2}, \\
& t_{12}=t_{21}=x y, \\
& t_{13}=t_{31}=x \frac{\partial \phi}{\partial x}+x u, \\
& t_{14}=t_{41}=x \frac{\partial \phi}{\partial y}+x v, \\
& t_{22}=y^{2}, \\
& t_{23}=t_{32}=y \frac{\partial \phi}{\partial x}+y u,
\end{aligned}
$$

$$
\begin{aligned}
& t_{24}=t_{42}=y \frac{\partial \phi}{\partial y}+y v \\
& t_{33}=\left(\frac{\partial \phi}{\partial x}\right)^{2}+2 u\left(\frac{\partial \phi}{\partial x}\right)+u^{2} \\
& t_{34}=t_{43}=\left(\frac{\partial \phi}{\partial x}\right)\left(\frac{\partial \phi}{\partial y}\right)+u \frac{\partial \phi}{\partial y}+v \frac{\partial \phi}{\partial x}+u v, \\
& t_{44}=\left(\frac{\partial \phi}{\partial y}\right)^{2}+2 v\left(\frac{\partial \phi}{\partial y}\right)+v^{2}
\end{aligned}
$$

with $\left(1 / P_{\mathrm{i}}\right) \int t_{m n} h_{\mathrm{j}}(x, y, u, v ; z) \mathrm{d} x \mathrm{~d} y \mathrm{~d} u \mathrm{~d} v=$ $\left\langle t_{m n}\right\rangle_{\mathrm{i}}, m, n=1, \ldots, 4$. Thus, for example, $\left\langle t_{13}\right\rangle_{\mathrm{i}}=$ $\left\langle t_{31}\right\rangle_{\mathrm{i}}=\langle x(\partial \phi / \partial x)\rangle_{\mathrm{i}}+\langle x u\rangle_{\mathrm{i}}$, and similarly for the other elements of matrix $\mathbf{T}$.

On the other hand, from direct application of Eq. (2) it can be shown (see Appendix) that the secondorder moments at the output of a PPT are given in terms of the moments at the input plane of the transmittance by the formulae

$$
\begin{aligned}
\left\langle x^{2}\right\rangle_{0}= & \left\langle x^{2}\right\rangle_{\mathrm{i}}, \\
\langle x y\rangle_{0}= & \langle x y\rangle_{\mathrm{i}}, \\
\langle x u\rangle_{\mathrm{o}}= & \left\langle x \frac{\partial \phi}{\partial x}\right\rangle_{\mathrm{i}}+\langle x u\rangle_{\mathrm{i}}, \\
\langle x v\rangle_{0}=\left\langle x \frac{\partial \phi}{\partial y}\right\rangle_{\mathrm{i}}+\langle x v\rangle_{\mathrm{i}}, & \\
\left\langle y^{2}\right\rangle_{\mathrm{o}}= & \left\langle y^{2}\right\rangle_{\mathrm{i}}, \\
\langle y u\rangle_{0}= & \left\langle y \frac{\partial \phi}{\partial x}\right\rangle_{\mathrm{i}}+\langle y u\rangle_{\mathrm{i}}, \\
\langle y v\rangle_{0}= & \left\langle y \frac{\partial \phi}{\partial y}\right\rangle_{\mathrm{i}}+\langle y v\rangle_{\mathrm{i}}, \\
\left\langle u^{2}\right\rangle_{0}= & \left\langle\left(\frac{\partial \phi}{\partial x}\right)^{2}\right\rangle_{\mathrm{i}}+\left\langle 2 u \frac{\partial \phi}{\partial x}\right\rangle_{\mathrm{i}}+\left\langle u^{2}\right\rangle_{\mathrm{i}}, \\
\langle u v\rangle_{0}= & \left\langle\left(\frac{\partial \phi}{\partial x}\right)\left(\frac{\partial \phi}{\partial y}\right)\right\rangle_{\mathrm{i}}+\left\langle u \frac{\partial \phi}{\partial y}\right\rangle_{\mathrm{i}}+\left\langle v \frac{\partial \phi}{\partial x}\right\rangle_{\mathrm{i}} \\
& +\langle u v\rangle_{\mathrm{i}}, \\
\left\langle u^{2}\right\rangle_{0}= & \left\langle\left(\frac{\partial \phi}{\partial y}\right)^{2}\right\rangle_{\mathrm{i}}+\left\langle 2 v \frac{\partial \phi}{\partial y}\right\rangle_{\mathrm{i}}+\left\langle v^{2}\right\rangle_{\mathrm{i}},
\end{aligned}
$$

But the above expressions are identical to those obtained from the substitution of the elements of matrix $T$ into the integral that appears in the right-hand side of Eq. (8). Consequently, Eq. (8) is fulfilled. 
It should be noted that matrix $\mathbf{M}_{\mathrm{PP}}$, as given by Eq. (10), depends on the reference coordinate systems only through the function $\phi(x, y)$. In other words, if the plate is placed rotated about the $z$-axis, the specific form of function $\phi(x, y)$ would, in general, change, but Eq. (10) is still valid.

In the particular case, $t(x, y)=\exp [-(i k / 2 f)$ $\left.\times\left(x^{2}+y^{2}\right)\right]$ (non-abcrrated thin lens), matrix $\mathbf{C}$ reduces to

$\mathbf{C}=\left(\begin{array}{cc}-1 / f & 0 \\ 0 & -1 / f\end{array}\right)$

in complete agreement with the well-known $A B C D$ matrix of such a lens. It is important to note that the propagation laws through PPTs must be written in the form given by Eqs. (7) and (8), instead of in the more usual form (valid for ordinary $A B C D$ optical elements only)

$\mathbf{F}_{0}=\mathbf{M F}_{1}$,

and

$\mathbf{S}_{0}=\mathbf{M S}_{\mathrm{i}} \mathbf{M}^{t}$,

where $\mathbf{M}$ now represents the matrix of the $A B C D$ system. Expressions (17) and (18) would not make sense if matrix $\mathbf{M}$ were directly substituted by $\mathbf{M}_{\mathrm{PPT}}$ in these equations.

Accordingly, the propagation laws of the intensity moments of beams propagating through cascaded optical systems containing PPTs must also be written as follows

$$
\begin{aligned}
\mathbf{F}_{\mathrm{o}} & =\frac{1}{P_{\mathrm{i}}} \int \mathbf{M}_{N} \mathbf{M}_{N-1} \ldots \mathbf{M}_{1} \hat{p} \\
& \times h_{\mathrm{i}}(x, y, u, v ; z) \mathrm{d} x \mathrm{~d} y \mathrm{~d} u \mathrm{~d} v
\end{aligned}
$$

and

$$
\begin{aligned}
\mathbf{S}_{\mathrm{o}} & =\frac{1}{P_{\mathrm{i}}} \int \mathbf{M}_{N} \mathbf{M}_{N-1} \ldots \mathbf{M}_{1} \hat{p} \hat{p}^{t} \mathbf{M}_{1}^{t} \ldots \mathbf{M}_{N-1}^{t} \mathbf{M}_{N}^{t} \\
& \times h_{\mathrm{i}}(x, y, u, v ; z) \mathrm{d} x \mathrm{~d} y \mathrm{~d} u \mathrm{~d} v
\end{aligned}
$$

where $\mathbf{M}_{j}, j=1, \ldots, N$, denote the matrices associated with the optical elements (including PPTs), multiplied in the same order as they are crossed by the beam.

\section{Application to quartic phase transmittances}

Use of matrix $\mathbf{M}_{\mathrm{PPT}}$ can significantly shorten the calculations (usually rather cumbersome) required to get the moments and other related parameters (e.g., beam quality) when a beam propagates through a PP'I. As a simple application of the above matrix, we will now consider the effect that quartic phase distortions produce in the intensity moments of general beams. Two cases of special interest will be analysed: (i) a single circular (rotationally symmetric) spherically aberrated lens, and (ii) two identical but orthogonal spherically aberrated cylindrical lenses in contact with one another. The changes generated in a number of beam quality parameters have recently been investigated [10]. In the following, by using matrix $\mathbf{M}_{\mathrm{PPT}}$, we will provide all second-order moments at the output of these kinds of quartic phase transmittances.

\subsection{Single lens}

A spherical aberration can be represented by means of the following transmittance function $[10,11]$.

$t(x, y)=\exp \left[i k a\left(x^{2}+y^{2}\right)^{2}\right]$,

where $a$ is a constant. Therefore, matrix $\mathbf{C}$ (see Eq. (11)) takes the form

$\mathbf{C}=\left(\begin{array}{cc}4 a\left(x^{2}+y^{2}\right) & 0 \\ 0 & 4 a\left(x^{2}+y^{2}\right)\end{array}\right)$.

If we assume, for simplicity, that $\langle x\rangle=\langle y\rangle=\langle u\rangle=$ $\langle v\rangle=0$ (this is simply equivalent to a shift of the coordinate system), direct application of Eqs. (8) and (10) then gives

$$
\begin{aligned}
\left\langle x^{2}\right\rangle_{\mathrm{o}}= & \left\langle x^{2}\right\rangle_{\mathrm{i}}, \\
\langle x y\rangle_{\mathrm{o}}= & \langle x y\rangle_{\mathrm{i}}, \\
\langle x u\rangle_{\mathrm{o}}= & \langle x u\rangle_{\mathrm{i}}+4 a\left\langle x^{4}\right\rangle_{\mathrm{i}}+4 a\left\langle x^{2} y^{2}\right\rangle_{\mathrm{i}}, \\
\langle x v\rangle_{\mathrm{o}}= & \langle x v\rangle_{\mathrm{i}}+4 a\left\langle x^{3} y\right\rangle_{\mathrm{i}}+4 a\left\langle x y^{3}\right\rangle_{\mathrm{i}}, \\
\left\langle y^{2}\right\rangle_{\mathrm{o}}= & \left\langle y^{2}\right\rangle_{\mathrm{i}}, \\
\langle y u\rangle_{\mathrm{o}}= & \langle y u\rangle_{\mathrm{i}}+4 a\left\langle x^{3} y\right\rangle_{\mathrm{i}}+4 a\left\langle x y^{3}\right\rangle_{\mathrm{i}}, \\
\langle y v\rangle_{\mathrm{o}}= & \langle y v\rangle_{\mathrm{i}}+4 a\left\langle x^{2} y^{2}\right\rangle_{\mathrm{i}}+4 a\left\langle y^{4}\right\rangle_{\mathrm{i}}, \\
\left\langle u^{2}\right\rangle_{\mathrm{o}}= & \left\langle u^{2}\right\rangle_{\mathrm{i}}+16 a^{2}\left(\left\langle x^{6}\right\rangle_{\mathrm{i}}+2\left\langle x^{4} y^{2}\right\rangle_{\mathrm{i}}+\left\langle x^{2} y^{4}\right\rangle_{\mathrm{i}}\right) \\
& +8 a\left(\left\langle x^{3} u\right\rangle_{\mathrm{i}}+\left\langle x u y^{2}\right\rangle_{\mathrm{i}}\right),
\end{aligned}
$$




$$
\begin{aligned}
\langle u v\rangle_{\mathrm{o}}= & \langle u v\rangle_{\mathrm{i}} \\
& +4 a\left(\left\langle x^{3} v\right\rangle_{\mathrm{i}}+\left\langle y^{3} u\right\rangle_{\mathrm{i}}+\left\langle x y^{2} v\right\rangle_{\mathrm{i}}+\left\langle x^{2} y u\right\rangle_{\mathrm{i}}\right) \\
& +16 a^{2}\left(2\left\langle x^{3} y^{3}\right\rangle_{\mathrm{i}}+\left\langle x^{5} y\right\rangle_{\mathrm{i}}+\left\langle x y^{5}\right\rangle_{\mathrm{i}}\right), \quad(23 \mathrm{i}) \\
\left\langle v^{2}\right\rangle_{\mathrm{o}}= & \left\langle v^{2}\right\rangle_{\mathrm{i}}+16 a^{2}\left(\left\langle y^{6}\right\rangle_{\mathrm{i}}+2\left\langle x^{2} y^{4}\right\rangle_{\mathrm{i}}+\left\langle x^{4} y^{2}\right\rangle_{\mathrm{i}}\right) \\
& +8 a\left(\left\langle y^{3} v\right\rangle_{\mathrm{i}}+\left\langle x^{2} y v\right\rangle_{\mathrm{i}}\right) .
\end{aligned}
$$

These expressions are consistent with the values of the beam quality parameters obtained in Ref. [10] for the special case $\langle x y\rangle_{\mathrm{i}}=0$.

\subsection{Two orthogonal cylindrical lenses}

In this case function $t(x, y)$ reads

$t(x, y)=\exp \left[i k a\left(x^{4}+y^{4}\right)\right]$.

Matrix $\mathbf{C}$ now becomes

$\mathbf{C}=\left(\begin{array}{cc}4 a x^{2} & 0 \\ 0 & 4 a y^{2}\end{array}\right)$

and the second-order intensity moments are

$$
\begin{aligned}
\left\langle x^{2}\right\rangle_{0}= & \left\langle x^{2}\right\rangle_{\mathrm{i}}, \\
\langle x y\rangle_{0}= & \langle x y\rangle_{\mathrm{i}}, \\
\langle x u\rangle_{0}= & \langle x u\rangle_{\mathrm{i}}+4 a\left\langle x^{4}\right\rangle_{\mathrm{i}}, \\
\langle x v\rangle_{0}= & \langle x v\rangle_{\mathrm{i}}+4 a\left\langle x y^{3}\right\rangle_{\mathrm{i}}, \\
\left\langle y^{2}\right\rangle_{\mathrm{o}}= & \left\langle y^{2}\right\rangle_{\mathrm{i}}, \\
\langle y u\rangle_{\mathrm{o}}= & \langle y u\rangle_{\mathrm{i}}+4 a\left\langle x^{3} y\right\rangle_{\mathrm{i}}, \\
\langle y v\rangle_{\mathrm{o}}= & \langle y v\rangle_{\mathrm{i}}+4 a\left\langle y^{4}\right\rangle_{\mathrm{i}}, \\
\left\langle u^{2}\right\rangle_{\mathrm{o}}= & \left\langle u^{2}\right\rangle_{\mathrm{i}}+16 a^{2}\left\langle x^{6}\right\rangle_{\mathrm{i}}+8 a\left\langle x^{3} u\right\rangle_{\mathrm{i}}, \\
\langle u v\rangle_{\mathrm{o}}= & \langle u v\rangle_{\mathrm{i}}+4 a\left(\left\langle x^{3} v\right\rangle_{\mathrm{i}}+\left\langle y^{3} v\right\rangle_{\mathrm{i}}\right) \\
& +16 a^{2}\left\langle x^{3} y^{3}\right\rangle_{\mathrm{i}}, \\
\left\langle v^{2}\right\rangle_{0}= & \left\langle v^{2}\right\rangle_{\mathrm{i}}+16 a^{2}\left\langle y^{6}\right\rangle_{\mathrm{i}}+8 a\left\langle y^{3} v\right\rangle_{\mathrm{i}} .
\end{aligned}
$$

As above, these expressions are consistent with the formulae handled in Ref. [ 10]. It is clear that, from an analytical point of view, the two-lens system involves much simpler expressions of the moments than the single-lens case.

\section{Acknowledgements}

The research work leading to this paper has been supported by the Comisión Interministerial de Ciencia y Tecnología of Spain, under Project No. TAP93-211, within the framework of EU-1269 Project.

\section{Appendix A}

In this appendix we will demonstrate Eqs. (15).

To begin with note that $\left\langle x^{2}\right\rangle,\left\langle y^{2}\right\rangle$ and $\langle x y\rangle$ are moments of spatial variables only, so that Eqs. (15a), (15b) and (15e) follow at once. On the other hand, by taking Eq. (9) into account, we have

$$
\begin{aligned}
\langle x u\rangle_{\mathrm{o}} & =\frac{1}{2 i k P_{\mathrm{i}}} \int g^{*} \frac{\partial g}{\partial x}-g \frac{\partial g^{*}}{\partial x} \mathrm{~d} x \mathrm{~d} y \\
= & \frac{1}{2 i k P_{\mathrm{i}}} \int f^{*} \frac{\partial f}{\partial x}-f \frac{\partial f^{*}}{\partial x}+2 i k \frac{\partial \phi}{\partial x}|f|^{2} \mathrm{~d} x \mathrm{~d} y \\
& =\langle x u\rangle_{\mathrm{i}}+\left\langle x \frac{\partial \phi}{\partial x}\right\rangle_{\mathrm{i}}
\end{aligned}
$$

which is identical to Eq. (15c). In a similar way Eqs. (15d), (15f) and (15g) can be proved. It can also be shown that

$$
\begin{aligned}
& \langle u v\rangle_{\mathrm{o}}= \\
& \frac{1}{2 k^{2} P_{\mathrm{i}}} \int\left[\left(\frac{\partial g^{*}}{\partial x}\right)\left(\frac{\partial g}{\partial y}\right)+\left(\frac{\partial g^{*}}{\partial y}\right)\left(\frac{\partial g}{\partial x}\right)\right] \mathrm{d} x \mathrm{~d} y \\
& \quad=\langle u v\rangle_{\mathrm{i}}+\left\langle\left(\frac{\partial \phi}{\partial x}\right)\left(\frac{\partial \phi}{\partial y}\right)\right\rangle_{\mathrm{i}} \\
& +\frac{i}{2 k P_{\mathrm{i}}} \int \frac{\partial \phi}{\partial y}\left(f \frac{\partial f^{*}}{\partial x}-f^{*} \frac{\partial f}{\partial x}\right) \mathrm{d} x \mathrm{~d} y \\
& +\frac{i}{2 k P_{\mathrm{i}}} \int \frac{\partial \phi}{\partial x}\left(f \frac{\partial f^{*}}{\partial y}-f^{*} \frac{\partial f}{\partial y}\right) \mathrm{d} x \mathrm{~d} y .
\end{aligned}
$$

But

$\left\langle u \frac{\partial \phi}{\partial y}\right\rangle=\frac{i}{2 k P_{\mathrm{i}}} \int \frac{\partial \phi}{\partial y}\left(f \frac{\partial f^{*}}{\partial x}-f^{*} \frac{\partial f}{\partial x}\right) \mathrm{d} x \mathrm{~d} y$.

and

$\left\langle v \frac{\partial \phi}{\partial x}\right\rangle=\frac{i}{2 k P_{\mathrm{i}}} \int \frac{\partial \phi}{\partial x}\left(f \frac{\partial f^{*}}{\partial y}-f^{*} \frac{\partial f}{\partial y}\right) \mathrm{d} x \mathrm{~d} y$.

Eq. (15i) then follows from the substitution of Eqs. (A3) and (A4) into Eq. (A2). 
Finally, since

$$
\begin{aligned}
& \left|\frac{\partial g}{\partial x}\right|^{2}=\left|\frac{\partial f}{\partial x}\right|^{2}+k^{2}\left(\frac{\partial \phi}{\partial x}\right)^{2}|f|^{2} \\
& +i k \frac{\partial \phi}{\partial x}\left(f \frac{\partial f^{*}}{\partial x}-f^{*} \frac{\partial f}{\partial x}\right) \mathrm{d} x d y,
\end{aligned}
$$

we have

$$
\begin{aligned}
& \left\langle u^{2}\right\rangle_{0}=\frac{1}{k^{2} P_{\mathrm{i}}} \int\left|\frac{\partial g}{\partial x}\right|^{2} \mathrm{~d} x \mathrm{~d} y \\
& =\left\langle u^{2}\right\rangle_{\mathrm{i}}+\left\langle\left(\frac{\partial \phi}{\partial x}\right)^{2}\right\rangle_{\mathrm{i}}+2\left\langle u\left(\frac{\partial \phi}{\partial x}\right)\right\rangle_{\mathrm{i}},
\end{aligned}
$$

which is identical to Eq. (15h). In a similar way Eq. (15j) can be proved.

\section{References}

[1] S. Lavi, R. Prochaska and E. Keren, Appl. Optics 27 (1988) 3696.

[2] A.E. Siegman, Proc. SPIE 1224 (1990) 2.

[3] M.J. Bastiaans, Optik 88 (1991) 163.

[4] J. Serna, R. Martínez-Herrero and P.M. Mejías, J. Opt. Soc. Am. A 8 (1991) 1094.

[5] H. Weber, Opt. Quantum. Electron. 24 (1992) 1027.

[6] R. Martínez-Herrero and P.M. Mejías, Optics Comm. 95 (1993) 18.

[7] R. Martínez-Herrero, P.M. Mejías and G. Piquero, Optics Lett. 17 (1992) 1650.

[8] L. Mandel, E. Wolf, J. Opt. Soc. Am. 66 (1976) 529.

[9] J. Serna, P.M. Mejías and R. Martínez-Herrero, Opt. Quantum Electron. 24 (1992) 873.

[10] R. Martínez-Herrero, G. Piquero and P.M. Mejías, Opt. Quantum Electron. 27 (1995) 173.

[11] A.E. Siegman, Appl. Optics 23 (1993) 5893.

[12] R. Martínez-Herrero, P.M. Mejías and H. Weber, Opt. Quantum Electron. 25 ( 1993 ) 423. 